\title{
Probing for massive stochastic gravitational-wave background with a detector network
}

\section{$\operatorname{AUTHOR}(S)$ :}

Nishizawa, Atsushi; Hayama, Kazuhiro

\section{CITATION:}

Nishizawa, Atsushi ...[et al]. Probing for massive stochastic gravitational-wave background with a detector network. Physical Review D 2013, 88(6): 064005.

ISSUE DATE:

2013-09-03

URL:

http://hdl.handle.net/2433/179287

RIGHT:

(C) 2013 American Physical Society 
PHYSICAL REVIEW D 88, 064005 (2013)

\title{
Probing for massive stochastic gravitational-wave background with a detector network
}

\author{
Atsushi Nishizawa ${ }^{1, *}$ and Kazuhiro Hayama ${ }^{2,3}$ \\ ${ }^{1}$ Department of Physics, Kyoto University, Kyoto 606-8502, Japan \\ ${ }^{2}$ Faculty of Science, Graduate School of Science, Osaka City University, 3-3-138 Sugimoto Sumiyoshi-ku, Osaka-shi 558-8585, Japan \\ ${ }^{3}$ TAMA Project, National Astronomical Observatory of Japan, Mitaka, Tokyo 181-8588, Japan
}

(Received 5 July 2013; published 3 September 2013)

\begin{abstract}
In a general metric theory of gravitation in four dimensions, six polarizations of a gravitational wave are allowed: two scalar and two vector modes, in addition to two tensor modes in general relativity. Such additional polarization modes appear due to additional degrees of freedom in modified gravity theories. Also, graviton mass, which could be different in each polarization, is another characteristic of modification of gravity. Thus, testing the existence of additional polarization modes and graviton mass can be a model-independent test of gravity theories. Here we extend the previous framework of correlation analysis of a gravitational-wave background to the massive case and show that a ground-based detector network can probe for massive stochastic gravitational waves with its mass around $\sim 10^{-14} \mathrm{eV}$. We also show that more than three detectors can cleanly separate the mixture of polarization modes in detector outputs and determine the graviton mass.
\end{abstract}

DOI: 10.1103/PhysRevD.88.064005

PACS numbers: 04.50.Kd, 04.80.Cc, 04.30.-w

\section{INTRODUCTION}

In a past decade, direct detection experiments of a gravitational wave $(\mathrm{GW})$ have been well developed. The next-generation kilometer-scale laser-interferometric GW detectors such as advanced LIGO (aLIGO) [1], advanced VIRGO (aVIRGO) [2], and KAGRA [3] are under construction and will start observation within the coming three to five years, aiming at the first detection of a GW. The GW observation not only brings us valuable information about astronomy and cosmology but also allows us to test a gravity theory.

If a gravity theory deviates from general relativity (GR), GWs are also modified in (i) the GW waveform, mainly phase evolution, (ii) the additional polarization modes, and (iii) the graviton mass or propagation speed (for a review, see Refs. [4-6]). The first one is associated with the dynamics of a compact binary. A gravity test in this direction has been investigated by many authors (see Ref. [6] and references therein). However, most works rely on the matched filtering technique [7], which requires accurate wave templates in a specific model of a gravity theory and is a model-dependent method. Some exceptions are modelindependent studies focusing on deviation from GR [8-13]. Secondly, additional polarization modes arise due to extra degrees of freedom appearing in a modified gravity theory. In GR, a GW has two tensor polarization modes (plus and cross modes), while in a general metric theory of gravitation, the GW is allowed to have at most six polarizations, including scalar and vector modes $[14,15]$. The number of polarizations in various gravity theories has been studied in Refs. [16-18], and the model-independent test has been proposed in Refs. [19-22]. In the third one,

*nishizawa@tap.scphys.kyoto-u.ac.jp graviton mass changes the propagation speed of a GW. Namely, the dispersion relation of a GW is modified, and a GW undergoes a mass-dependent phase evolution during its propagation. Constraint on graviton mass from the observation of compact binary coalescences was originally proposed in Ref. [23] and later forecasted with more accurate GW waveforms including spin-orbit and spinspin couplings and spin precession effects [24,25] (see also the recent paper [26] and references therein). Also, mass constraint will be obtained from the observation of a gravitational-wave background (GWB) in a pulsar timing array [27]. Since the polarization and massive graviton tests do not demand one to know neither dynamics of astronomical objects nor exact waveforms, we are able to perform a model-independent test of gravity.

Here we consider a stochastic GWB, which is the incoherent superposition of gravitational waves produced by many unresolved astronomical sources or by inflation and reheating in the early Universe. The sensitivity of a GW detector pair to a GWB with scalar polarization has been investigated in Refs. [28,29]. However, their studies assumed the existence of a single polarization (scalar) mode. Thus, it is not manifest how the mixing with other polarization modes (tensor or vector) affects the detector sensitivity to the scalar mode and the mode separability. Previously, we studied the detection and separation of scalar-, vector-, and tensor-polarization modes of a GWB using a network of ground-based laser interferometers [19] (with the pulsar timing array, see Ref. [30]). We found that with the correlation signals obtained from more than three detectors, the scalar, vector, and tensor modes of a GWB can be cleanly separated and detected without degeneracy between polarizations. The mode separation has been extensively studied in Ref. [20], varying the detector relative distance and orientation and the number of detectors. 
Then we found the general conditions required for successful mode separation and that a current network of groundbased detectors was accidentally nearly optimal. However, the previous studies assumed that the graviton was massless. It is likely that the graviton has mass if gravity is modified and a GW has an additional polarization mode. In fact, the tensor graviton is massless, and the scalar graviton is massive in $f(R)$ gravity [31,32] and the scalar-tensor theory [33]. In this paper, we further extend our previous formalism to a massive GWB containing scalar, vector, and tensor polarizations and investigate what range of graviton mass is searched by ground-based detectors and how accurately the graviton mass is determined.

The organization of this paper is as follows. In Sec. II, we briefly review the formulation of a GWB with scalar, vector, and tensor polarizations and extend it to the massive case. We also mention current constraints on graviton mass. In Sec. III, the cross-correlation analysis of a GWB is extended to the massive case, focusing on how the standard analysis method in the massless case is altered. Sec. IV is the main part of this paper and describes parameter estimation accuracy based on the Fisher information matrix. In Sec. V, we discuss the parameter estimation accuracy in a more practical situation, that is, in a mixture of three polarization modes of a GWB. We devote Sec. VI to the summary of this paper.

\section{GW IN MODIFIED GRAVITY THEORIES}

\section{A. GW polarization modes}

In general, a metric gravity theory in four dimensions allows at most six polarization modes of a GW [14,34]. Let us define a wave orthonormal coordinate that is constructed by a unit vector $\hat{\Omega}$ directed to the propagation direction of a GW and two unit vectors $\hat{e}_{\theta}$ and $\hat{e}_{\phi}$ orthogonal to $\hat{\Omega}$ and each other. With these vectors, the polarization modes are defined as

$$
\begin{gathered}
\mathbf{e}^{+} \equiv \hat{e}_{\theta} \otimes \hat{e}_{\theta}-\hat{e}_{\phi} \otimes \hat{e}_{\phi}, \\
\mathbf{e}^{\times} \equiv \hat{e}_{\theta} \otimes \hat{e}_{\phi}+\hat{e}_{\phi} \otimes \hat{e}_{\theta}, \\
\mathbf{e}^{b} \equiv \hat{e}_{\theta} \otimes \hat{e}_{\theta}+\hat{e}_{\phi} \otimes \hat{e}_{\phi}, \\
\mathbf{e}^{\ell} \equiv \sqrt{2} \hat{\Omega} \otimes \hat{\Omega} \\
\mathbf{e}^{x} \equiv \hat{e}_{\theta} \otimes \hat{\Omega}+\hat{\Omega} \otimes \hat{e}_{\theta}, \\
\mathbf{e}^{y} \equiv \hat{e}_{\phi} \otimes \hat{\Omega}+\hat{\Omega} \otimes \hat{e}_{\phi},
\end{gathered}
$$

where the symbol $\otimes$ denotes a tensor product. The,$+- \times-$, $b-, \ell-, x-$, and $y$-polarization modes are called the plus, cross, breathing, longitudinal, vector- $x$, and vector- $y$ modes, respectively. Each polarization mode is orthogonal to one another and is normalized so that $e_{i j}^{A} e_{A^{\prime}}^{i j}=2 \delta_{A A^{\prime}}, A$,
$A^{\prime}=+, \times, b, \ell, x, y$. According to rotation symmetry about the propagation axis of a $\mathrm{GW}$, the + and $\times$ modes are identified with tensor-type (spin-2) GWs, the $x$ and $y$ modes are vector-type (spin-1) GWs, and the $b$ and $\ell$ modes are scalar-type (spin-0) GWs. Note that the breathing and longitudinal modes are not traceless, in contrast to the ordinary plus- and cross-polarization modes in GR.

A GW with the six polarizations is expressed as

$$
h_{i j}(t, \vec{X}, \hat{\Omega})=\sum_{A} h_{A}(t, \vec{X}) e_{i j}^{A}(\hat{\Omega}),
$$

where $A=+, \times, b, \ell, x, y$. The antenna pattern functions for each polarization mode $[19,35]$ are defined as

$$
F_{A}(\hat{\Omega}) \equiv D^{i j} e_{i j}^{A}(\hat{\Omega}), \quad \mathbf{D} \equiv \frac{1}{2}[\hat{u} \otimes \hat{u}-\hat{v} \otimes \hat{v}],
$$

where the unit vectors $\hat{u}$ and $\hat{v}$ are along two arms of a laser-interferometric detector, and $\mathbf{D}$ is a so-called detector tensor, which describes the response of the laserinterferometric detector to the polarization tensors. With these antenna pattern functions, a GW signal from a detector is written as

$$
h(t, \vec{X}, \hat{\Omega})=\sum_{A} h_{A}(t, \vec{X}) F_{A}(\hat{\Omega}) .
$$

For the derivation and the explicit expressions of the antenna pattern functions, see Ref. [19].

\section{B. Massive gravitational waves}

Another characteristic of a GW in modified gravity is graviton mass. If graviton possesses the mass $m_{g}$, its dispersion relation is altered as

$$
\omega^{2}=m_{g}^{2}+k^{2},
$$

where $\omega$ is the angular frequency and $k$ is the wave number. Consequently, gravitons propagate with the group velocity slower than the speed of light,

$$
v_{g}\left(\omega ; m_{g}\right) \equiv \frac{d \omega}{d k}=\sqrt{1-\frac{m_{g}^{2}}{\omega^{2}}},
$$

and the arrival time of the GW from a point source at each detector is delayed. On the other hand, the phase velocity is faster than the speed of light:

$$
v_{p}\left(\omega ; m_{g}\right) \equiv \frac{\omega}{k}=\left(\sqrt{1-\frac{m_{g}^{2}}{\omega^{2}}}\right)^{-1} .
$$

Then the plane wave solution of a GW is modified as

$$
e^{i(\omega t-\vec{k} \cdot \vec{X})}=e^{i \omega\left[t-\hat{\Omega} \cdot \vec{X} / v_{p}\left(\omega ; m_{g}\right)\right]},
$$

replacing the speed of light $c$ in GR with $v_{p}$. One may regard the change of the phase velocity from $c$ as the change of the effective detector distance from the origin, $\vec{x} / c \rightarrow \vec{x} / v_{p}$. Since $c<v_{p}$, the effective detector distance for a massive $\mathrm{GW}$ is smaller. 
For a stochastic GW background, the modification of the group velocity is not relevant, but the phase velocity plays an important role. The stochastic GW signal observed at a position $\vec{x}$ at time $t$ is written as

$h(t, \vec{X})=\sum_{A} \int_{S^{2}} d \hat{\Omega} \int_{-\infty}^{\infty} d f \tilde{h}_{A}(f, \hat{\Omega}) e^{2 \pi i f\left(t-\hat{\Omega} \cdot \vec{X} / v_{p}\right)} F_{A}(\hat{\Omega})$,

where $\tilde{h}_{A}(f, \hat{\Omega})$ is the Fourier transform of the GW amplitude in each polarization mode and the frequency is $f=\omega /(2 \pi)$. The Fourier transform of Eq. (12) is given by

$\tilde{h}(f, \vec{x})=\sum_{A} \int_{S^{2}} d \hat{\Omega} \tilde{h}_{A}(f, \hat{\Omega}) e^{-2 \pi i f \hat{\Omega} \cdot \vec{X} / v_{p}} F_{A}(\hat{\Omega})$.

Another consequence of the dispersion relation is the existence of the lowest angular frequency of a GW:

$$
\omega_{\text {min }}=m_{g} \approx 6.58 \times 10^{-14}\left(\frac{f_{g}}{100 \mathrm{~Hz}}\right) \mathrm{eV},
$$

where $f_{g}$ is the frequency corresponding to $m_{g}$. This is because below the frequency $\omega=m_{g}$, the wave number in the dispersion relation becomes imaginary and GWs become unstable. The reason is also understood directly from the definitions of graviton phase velocity in Eq. (10) and the plane wave solution in Eq. (11). As a result, a GW spectrum has a steep cutoff at the low-frequency side, independent of how the GW is generated.

\section{Stochastic GW spectrum}

In this paper, we assume that a stochastic GWB is (i) isotropic, (ii) independently polarized, (iii) stationary, and (iv) Gaussian, as discussed in detail in Ref. [36]. In this case, all the statistical properties of the GWB are characterized by

$$
\begin{aligned}
& \left\langle\tilde{h}_{A}^{*}(f, \hat{\Omega}) \tilde{h}_{A^{\prime}}\left(f^{\prime}, \hat{\Omega}^{\prime}\right)\right\rangle \\
& \quad=\delta\left(f-f^{\prime}\right) \frac{1}{4 \pi} \delta^{2}\left(\hat{\Omega}, \hat{\Omega}^{\prime}\right) \delta_{A A^{\prime}} \frac{1}{2} S_{h}^{A}(|f|),
\end{aligned}
$$

where $\delta^{2}\left(\hat{\Omega}, \hat{\Omega}^{\prime}\right) \equiv \delta\left(\phi-\phi^{\prime}\right) \delta\left(\cos \theta-\cos \theta^{\prime}\right)$ and $\langle\cdots\rangle$ denotes the ensemble average. $S_{h}^{A}(f)$ is the one-sided power spectral density of each polarization mode.

Conventionally, the amplitude of GWB for each polarization is characterized by an energy density per logarithmic frequency bin normalized by the critical energy density of the Universe:

$$
\Omega_{\mathrm{gw}}^{A}(f) \equiv \frac{1}{\rho_{c}} \frac{d \rho_{\mathrm{gw}}^{A}(f)}{d \ln f},
$$

where $\rho_{c}=3 H_{0}^{2} / 8 \pi G$ and the Hubble constant is $H_{0}=100 h_{0} \mathrm{~km} \mathrm{~s}^{-1} \mathrm{Mpc}^{-1}$. $\Omega_{\mathrm{gw}}(f)$ is related to $S_{h}(f)$ by $[36,37]$

$$
\Omega_{\mathrm{gw}}^{A}(f)=\left(\frac{2 \pi^{2}}{3 H_{0}^{2}}\right) f^{3} S_{h}^{A}(f)
$$

Note that the above definition is different from that in the literature [36,37] by a factor of 2 since it is defined for each polarization.

We assume that the + and $\times$ modes are not polarized (the detectability of circular polarizations in the polarized case has been discussed in Refs. [38-41]). We also assume that the $x$ and $y$ modes are not polarized. In most of the cosmological scenarios, these assumptions are valid. Then the GWB energy density of the tensor, vector, and scalar modes can be written as

$$
\begin{gathered}
\Omega_{\mathrm{gw}}^{T} \equiv \Omega_{\mathrm{gw}}^{+}+\Omega_{\mathrm{gw}}^{\times} \quad\left(\Omega_{\mathrm{gw}}^{+}=\Omega_{\mathrm{gw}}^{\times}\right), \\
\Omega_{\mathrm{gw}}^{V} \equiv \Omega_{\mathrm{gw}}^{x}+\Omega_{\mathrm{gw}}^{y} \quad\left(\Omega_{\mathrm{gw}}^{x}=\Omega_{\mathrm{gw}}^{y}\right), \\
\Omega_{\mathrm{gw}}^{S} \equiv \frac{1}{3}\left(\frac{1+2 \kappa}{1+\kappa}\right)\left(\Omega_{\mathrm{gw}}^{b}+\Omega_{\mathrm{gw}}^{\ell}\right),
\end{gathered}
$$

where the ratio of the energy density in the longitudinal mode to that in the breathing mode is characterized by the parameter $\kappa \equiv \Omega_{\mathrm{gw}}^{\ell} / \Omega_{\mathrm{gw}}^{b}$. However, we cannot determine $\kappa$ with a GW observation because the antenna pattern functions for both modes scale just by a factor of $\sqrt{2}$ and are completely degenerated. If a model of gravity theory is specified, $\kappa$ can be calculated theoretically and one can know the energy densities in the breathing and longitudinal modes from $\mathrm{GW}$ observation. Our definition of $\Omega_{\mathrm{gw}}^{S}$ is just for simplification of the formulation below. One should keep in mind that $\Omega_{\mathrm{gw}}^{S}$ does not exactly correspond to the physical energy density in the scalar mode but differs from the actual value up to a factor of 3 , depending on the ratio $\kappa$.

In this paper, we adopt the following parametrization for the spectral shape of a GWB:

$$
\Omega_{\mathrm{gw}}^{A}(f)=\Omega_{\mathrm{gw}, 0}^{A} s(f)\left(\frac{f}{f_{0}}\right)^{n_{t}^{A}} \Theta\left[f-f_{g}^{A}\right],
$$

where $\Theta[\cdot]$ is a step function. The shape function $s(f)$ strongly depends on the concrete generation mechanism of the GWB and the later expansion history of the Universe. In the standard cosmology with massless tensor gravitons, the GWB spectrum produced in slow-roll inflation has a nearly flat spectrum [42]. On the contrary, in massive gravity theory, a GWB spectrum from de Sitter inflation has an extremely sharp peak at $f=f_{g}^{A}$ when plotted as a function of frequency [43]. If detected, one could determine graviton mass very accurately from the peak frequency. However, its detectability strongly depends on the frequency resolution of a detector. According to the estimation with realistic observation time in Ref. [43], the peak would be smoothed in the frequency band of ground-based GW detectors, $\sim 100 \mathrm{~Hz}$ and difficult to detect. Our purpose in this paper is not to constrain a specific gravity theory but forecast the detectability of graviton mass with a detector network as general 
as possible. For this reason, hereafter we assume $s(f)=1$ for simplicity. In this case, the unknown parameters are the amplitude of the spectrum $\Omega_{\mathrm{gw}, 0}^{A}$, spectral index $n_{t}^{A}$, and mass of a graviton $m_{g}^{A}$ or equivalently corresponding frequency $f_{g}^{A}$ of each polarization mode. Since three polarization modes are in general independent of each other, we have nine parameters in total.

\section{Constraints on graviton mass}

Currently, graviton mass has been constrained by several observations of galaxies [44], the Solar System [45], and binary pulsars [46] (for a summary, see Ref. [26] and references therein). These limits are so tight that we can set graviton mass to zero in searching for stochastic GWs in the observational frequency band of ground-based detectors around $\sim 100 \mathrm{~Hz}$. In other words, there is no low-frequency cutoff of a GW spectrum and no difference from a massless case. However, the constraints from the Galaxy and the Solar System have been obtained from the observations in static gravitational fields and cannot be applied directly to GWs, while only the mass limit from binary pulsars comes from dynamical gravitational fields. According to the work by Finn and Sutton [46], the limit on the mass of gravitons is $m_{g}<7.6 \times 10^{-20} \mathrm{eV}$. From Eq. (14), this dynamical bound is far below the observational frequency of ground-based detectors. However, the bound can be applied to a tensor mode and not to scalar and vector modes. If there are scalar and vector degrees of freedom in a GW sector and their masses are so light that GWs are emitted by the binary pulsar, they would extract additional energy from the binary pulsars and the orbit decays faster than predicted in GR. The absence of such an observation means that their possible mass has to be large enough to suppress gravitational radiation if the additional modes exist. Therefore, graviton masses of scalar and vector GWs could be large enough to be detected with advanced detectors on Earth without contradicting with current observations.

On the other hand, if the suppression mechanism of the fifth force, such as the chameleon mechanism [47,48], works in order to elude the observational constraint from the Solar System [49], then scalar and vector gravitons could be much more massive beyond the reach of groundbased detectors in the high-density environment of matter. Also, another suppression mechanism called the Vainshtein mechanism [50,51] might conceal the deviation from GR. Additional degrees of freedom in gravity would alter the strength of gravity at cosmological scales and the growth of large-scale structure. This quasistatic perturbative regime of gravity has been tested with current cosmological observations and will be tested in the future with GWs $[52,53]$. However, no experiment has ever confirmed whether such suppression mechanisms work well in the perturbative and dynamical regime of gravity, particularly in GW itself. Thus, it would be worth searching for graviton mass experimentally with GW detectors in order to directly test the suppression mechanisms of the fifth force.

\section{CROSS-CORRELATION ANALYSIS}

We focus on a stochastic GWB and discuss cross correlation between a pair of detectors. To distinguish the GWB signal from stochastic detector noise independent in each detector, one has to correlate signals between two detectors. The correlation analysis has been well developed by several authors $[36,54,55]$. In this section, we will extend the formulation to massive GWs with tensor, vector, and scalar modes.

\section{A. Formulation}

Let us consider the outputs of a detector, $s(t)=h(t)+$ $n(t)$, where $h(t)$ and $n(t)$ are the GW signal and the noise of a detector. We assume that the amplitude of the GWB is much smaller than the detector noise. The cross-correlation signal $Y$ between two detectors is defined as

$$
Y \equiv \int_{-T / 2}^{T / 2} d t \int_{-T / 2}^{T / 2} d t^{\prime} s_{I}(t) s_{J}\left(t^{\prime}\right) Q\left(t-t^{\prime}\right)
$$

where $s_{I}$ and $s_{J}$ are outputs from the $I$ th and $J$ th detectors and $T$ is the observation time. $Q\left(t-t^{\prime}\right)$ is a filter function, which is later introduced so that the signal-to-noise ratio (SNR) is maximized. In the absence of intrinsic noise correlation between detectors, the ensemble average of the correlation signal $\mu \equiv\langle Y\rangle$ has a contribution only from GW signals. The derivation for massless GWs in a tensor-polarization mode has been given in Ref. [36]. The formulation has been extended to massless GWs with tensor, vector, and scalar modes in Ref. [19]. Further extension to massive gravitons with the three polarization modes is straightforward since the difference is merely replacing $c$ in the massless case with $v_{p}(f)$ as in Eq. (13). Tracing the standard procedure of the derivation, we obtain the following form:

$$
\mu=\frac{3 H_{0}^{2}}{20 \pi^{2}} T \sum_{A} \int_{-\infty}^{\infty} d f|f|^{-3} \gamma_{I J}^{A}(f) \Omega_{\mathrm{gw}}^{A}(f) \tilde{Q}(f) .
$$

In the above equation, we defined the overlap reduction functions

$$
\begin{aligned}
\gamma_{I J}^{T}\left(f ; m_{g}^{T}\right) \equiv & \frac{5}{2} \int_{S^{2}} \frac{d \hat{\Omega}}{4 \pi}\left(F_{I}^{+} F_{J}^{+}+F_{I}^{\times} F_{J}^{\times}\right) \\
& \times \exp \left[i \frac{2 \pi f \hat{\Omega} \cdot \Delta \vec{X}}{v_{p}\left(f ; m_{g}^{T}\right)}\right], \\
\gamma_{I J}^{V}\left(f ; m_{g}^{V}\right) \equiv & \frac{5}{2} \int_{S^{2}} \frac{d \hat{\Omega}}{4 \pi}\left(F_{I}^{x} F_{J}^{x}+F_{I}^{y} F_{J}^{y}\right) \\
& \times \exp \left[i \frac{2 \pi f \hat{\Omega} \cdot \Delta \vec{X}}{v_{p}\left(f ; m_{g}^{V}\right)}\right],
\end{aligned}
$$




$$
\begin{aligned}
\gamma_{I J}^{S}\left(f ; m_{g}^{S}\right) \equiv & \frac{15}{1+2 \kappa} \int_{S^{2}} \frac{d \hat{\Omega}}{4 \pi}\left(F_{I}^{b} F_{J}^{b}+\kappa F_{I}^{\ell} F_{J}^{\ell}\right) \\
& \times \exp \left[i \frac{2 \pi f \hat{\Omega} \cdot \Delta \vec{X}}{v_{p}\left(f ; m_{g}^{S}\right)}\right],
\end{aligned}
$$

where $\Delta \vec{X} \equiv \vec{X}_{I}-\vec{X}_{J}$. The overlap reduction functions represent how much the degree of correlation between the detectors in the GW signal is preserved and are normalized so that they give unity in the low-frequency limit for a coaligned detector pair.

The variance of the correlation signal is calculated by assuming that the noises in two detectors do not correlate at all and that the magnitude of the GW signal is much smaller than that of noise. Again following the standard procedure in Ref. [36], we obtain the variance of the correlation signal

$$
\begin{aligned}
\sigma^{2} & \equiv\left\langle Y^{2}\right\rangle-\langle Y\rangle^{2} \approx\left\langle Y^{2}\right\rangle \\
& =\frac{T}{4} \int_{-\infty}^{\infty} d f P_{I}(|f|) P_{J}(|f|)|\tilde{Q}(f)|^{2},
\end{aligned}
$$

where the one-sided power spectrum density of noise is defined by

$$
\left\langle\tilde{n}_{I}^{*}(f) \tilde{n}_{I}\left(f^{\prime}\right)\right\rangle \equiv \frac{1}{2} \delta\left(f-f^{\prime}\right) P_{I}(|f|) .
$$

Now we can determine the form of the optimal filter $\tilde{Q}(f)$ and derive the SNR formula. Equations (23) and (27) are expressed more simply, using an inner product

$$
(A, B) \equiv \int_{-\infty}^{\infty} d f A^{*}(f) B(f) P_{I}(|f|) P_{J}(|f|),
$$

as

$$
\begin{gathered}
\mu=\frac{3 H_{0}^{2}}{20 \pi^{2}} T\left(\tilde{Q}, \sum_{A} \frac{\gamma_{I J}^{A}(|f|) \Omega_{\mathrm{gw}}^{A}(|f|)}{|f|^{3} P_{I}(|f|) P_{J}(|f|)}\right), \\
\sigma^{2} \approx \frac{T}{4}(\tilde{Q}, \tilde{Q}) .
\end{gathered}
$$

From Eqs. (28) and (29), the SNR for GWB is defined as $\mathrm{SNR} \equiv \mu / \sigma$. Therefore, the optimal filter function and the SNR turn out to be

$\mathrm{SNR}=\frac{3 H_{0}^{2}}{10 \pi^{2}} \sqrt{T}\left[\int_{-\infty}^{\infty} d f \frac{\left\{\sum_{A} \gamma_{I J}^{A}(|f|) \Omega_{\mathrm{gw}}^{A}(|f|)\right\}^{2}}{f^{6} P_{I}(|f|) P_{J}(|f|)}\right]^{1 / 2}$,

and

$$
\tilde{Q}(f)=K \sum_{A} \frac{\gamma_{I J}^{A}(f) \Omega_{\mathrm{gw}}^{A}(|f|)}{|f|^{3} P_{I}(|f|) P_{J}(|f|)},
$$

with an arbitrary normalization factor $K$.

\section{B. Overlap reduction function}

The overlap reduction functions in Eqs. (24)-(26) can be written in a more convenient form by performing the angular integrals after expanding with tensorial bases. These expressions are basically the same as those in the massless case except for the replacement of $c$ with $v_{p}$. However, we summarize the expressions here for their later use.

We introduce the coordinate system on Earth shown in Fig. 1. The relative location and orientation of the two detectors are characterized by the three parameters $\beta, \sigma_{1}, \sigma_{2}$. The $\beta$ is the separation angle between two detectors measured from the center of Earth. The angles $\sigma_{1}$ and $\sigma_{2}$, are the orientations of the bisector of two arms of each detector measured in a counterclockwise manner relative to the great circle connecting the two detectors. The distance between the two detectors is

$$
|\Delta \mathbf{X}|=2 R_{E} \sin \frac{\beta}{2}
$$

where $R_{E}$ is the radius of Earth and we use $R_{E}=$ $6371 \mathrm{~km}$. Defining new parameters $\sigma_{+} \equiv\left(\sigma_{1}+\sigma_{2}\right) / 2$, $\sigma_{-} \equiv\left(\sigma_{1}-\sigma_{2}\right) / 2$, and

$$
\alpha(f) \equiv \frac{2 \pi f|\Delta \vec{X}|}{v_{p}\left(f ; m_{g}\right)}
$$

instead of frequency $f$, the overlap reduction functions in Eqs. (24)-(26) can be reduced in this coordinate system to

(i) tensor mode

$$
\begin{aligned}
\gamma^{T}\left(\alpha, \beta, \sigma_{+}, \sigma_{-}\right)= & \Theta_{T+}(\alpha, \beta) \cos \left(4 \sigma_{+}\right) \\
& +\Theta_{T-}(\alpha, \beta) \cos \left(4 \sigma_{-}\right),
\end{aligned}
$$

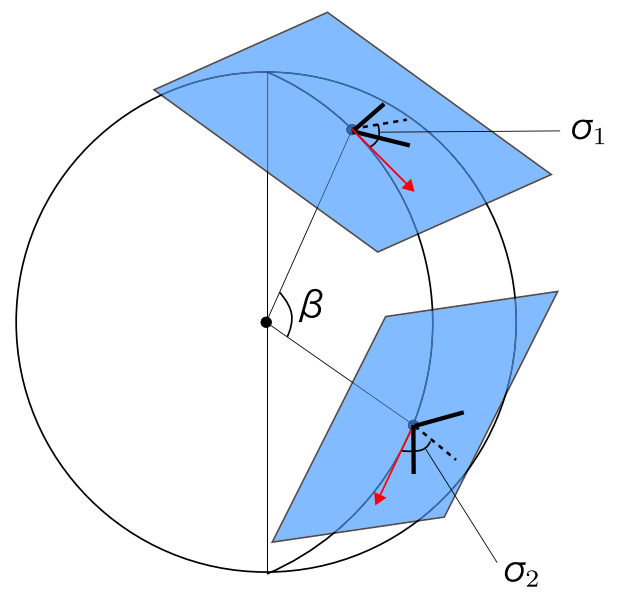

FIG. 1 (color online). Coordinate system on Earth for a detector pair. 
ATSUSHI NISHIZAWA AND KAZUHIRO HAYAMA

PHYSICAL REVIEW D 88, 064005 (2013)
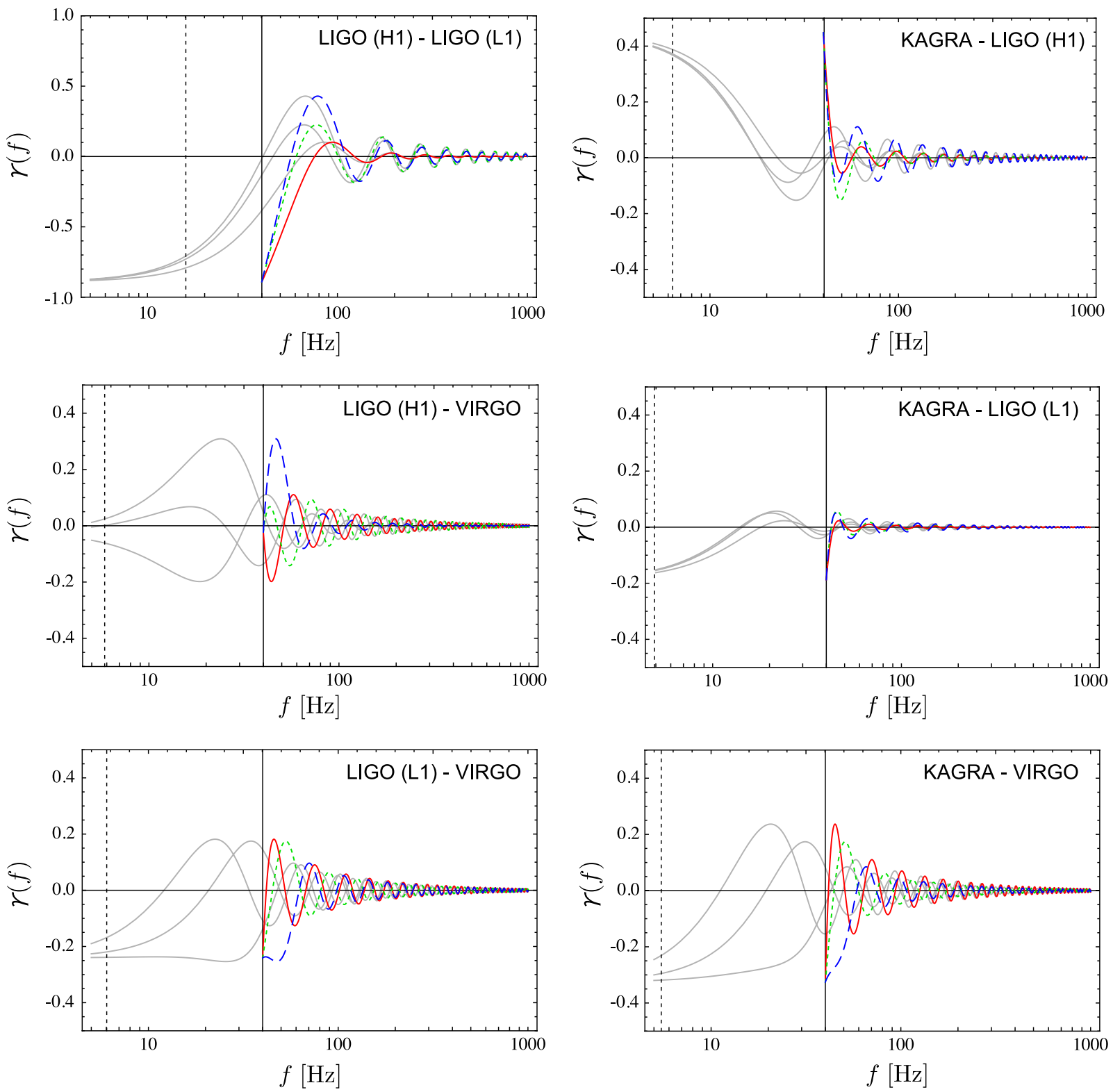

FIG. 2 (color online). Overlap reduction functions for real-detector pairs on Earth. The frequency corresponding to graviton mass is set to $f_{g}=40 \mathrm{~Hz}\left(m_{g} \approx 2 \times 10^{-14} \mathrm{eV}\right)$ (vertical solid line) for illustration. The characteristic frequency $f_{c}$, which is relevant for a massless overlap reduction function, is also shown (vertical dashed line). Each curve shows the tensor mode (red, solid), vector mode (green, dotted), and scalar mode (blue, dashed). The overlap reduction functions for the massless graviton case are also shown with monochromatic curves just for reference.

$$
\begin{aligned}
\Theta_{T+}(\alpha, \beta) \equiv & -\left(\frac{3}{8} j_{0}-\frac{45}{56} j_{2}+\frac{169}{896} j_{4}\right) \\
& +\left(\frac{1}{2} j_{0}-\frac{5}{7} j_{2}-\frac{27}{224} j_{4}\right) \cos \beta \\
& -\left(\frac{1}{8} j_{0}+\frac{5}{56} j_{2}+\frac{3}{896} j_{4}\right) \cos 2 \beta, \\
\Theta_{T-}(\alpha, \beta) \equiv & \left(j_{0}+\frac{5}{7} j_{2}+\frac{3}{112} j_{4}\right) \cos ^{4}\left(\frac{\beta}{2}\right),
\end{aligned}
$$

(ii) vector mode

$$
\begin{aligned}
\gamma^{V}\left(\alpha, \beta, \sigma_{+}, \sigma_{-}\right)= & \Theta_{V+}(\alpha, \beta) \cos \left(4 \sigma_{+}\right) \\
& +\Theta_{V-}(\alpha, \beta) \cos \left(4 \sigma_{-}\right), \\
\Theta_{V+}(\alpha, \beta) \equiv & -\left(\frac{3}{8} j_{0}+\frac{45}{112} j_{2}-\frac{169}{224} j_{4}\right) \\
& +\left(\frac{1}{2} j_{0}+\frac{5}{14} j_{2}+\frac{27}{56} j_{4}\right) \cos \beta \\
& -\left(\frac{1}{8} j_{0}-\frac{5}{112} j_{2}-\frac{3}{224} j_{4}\right) \cos 2 \beta,
\end{aligned}
$$




$$
\Theta_{V-}(\alpha, \beta) \equiv\left(j_{0}-\frac{5}{14} j_{2}-\frac{3}{28} j_{4}\right) \cos ^{4}\left(\frac{\beta}{2}\right),
$$

(iii) scalar mode

$$
\begin{aligned}
\gamma^{S}\left(\alpha, \beta, \sigma_{+}, \sigma_{-}\right)= & \Theta_{S+}(\alpha, \beta) \cos \left(4 \sigma_{+}\right) \\
& +\Theta_{S-}(\alpha, \beta) \cos \left(4 \sigma_{-}\right),
\end{aligned}
$$

$$
\begin{aligned}
\Theta_{S+}(\alpha, \beta) \equiv & -\left(\frac{3}{8} j_{0}+\frac{45}{56} j_{2}+\frac{507}{448} j_{4}\right) \\
& +\left(\frac{1}{2} j_{0}+\frac{5}{7} j_{2}-\frac{81}{112} j_{4}\right) \cos \beta \\
& -\left(\frac{1}{8} j_{0}-\frac{5}{56} j_{2}+\frac{9}{448} j_{4}\right) \cos 2 \beta,
\end{aligned}
$$

$$
\Theta_{S-}(\alpha, \beta) \equiv\left(j_{0}-\frac{5}{7} j_{2}+\frac{9}{56} j_{4}\right) \cos ^{4}\left(\frac{\beta}{2}\right)
$$

where $j_{n}(\alpha)$ is the spherical Bessel function with the argument $\alpha$.

We plot the overlap reduction functions in Fig. 2 for the existing detector pairs on Earth, whose relative coordinates $\left(\beta, \sigma_{+}, \sigma_{-}\right)$are listed in Table I.

Let us begin with the explanation for the massless case. The overlap reduction functions start to oscillate and decay rapidly above the characteristic frequency given by $f_{c} \equiv c /(2 \pi|\Delta \mathbf{X}|)$. At low frequencies, the functions approach constant values, which are determined by the relative orientations of the detector pair. The difference between the polarization modes starts to appear at around the characteristic frequency. Mathematically, this is because in Eqs. (34)-(41) the coefficients of $j_{0}$ in the overlap reduction functions are exactly the same, while the coefficients of $j_{2}$ and $j_{4}$ are different. To this end, at the low-frequency limit $\left(j_{0} \rightarrow 1\right.$, $j_{2} \rightarrow 0$, and $j_{4} \rightarrow 0$ ) all the overlap reduction functions degenerate.

TABLE I. Relative positions and orientations of detector pairs on Earth (in units of degrees) and the separation between the two detectors and the characteristic frequency of the overlap reduction function.

\begin{tabular}{lcrccc}
\hline \hline Detector pair & $\beta$ & \multicolumn{1}{c}{$\sigma_{+}$} & $\sigma_{-}$ & Separation $(\mathrm{km})$ & $f_{c}(\mathrm{~Hz})$ \\
\hline K-H & 72.4 & 25.6 & 89.1 & $7.52 \times 10^{3}$ & 6.3 \\
K-L & 99.2 & 68.1 & 42.4 & $9.71 \times 10^{3}$ & 4.8 \\
K-V & 86.6 & 5.6 & 28.9 & $8.74 \times 10^{3}$ & 5.4 \\
H-L & 27.2 & 62.2 & 45.3 & $3.00 \times 10^{3}$ & 16 \\
H-V & 79.6 & 55.1 & 61.1 & $8.16 \times 10^{3}$ & 5.7 \\
L-V & 76.8 & 83.1 & 26.7 & $7.91 \times 10^{3}$ & 6.0 \\
\hline \hline
\end{tabular}

On the other hand, in the massive case, the overlap reduction functions have a cutoff at the frequency $f_{g}$, at which the phase velocity diverges and $\alpha$ is zero. At the intermediate frequencies $f>f_{g}$, the detector distance $|\Delta \vec{X}|$ in $\alpha$ is effectively reduced because $|\Delta \vec{X}| / c$ in the massless case is replaced with $|\Delta \vec{X}| / v_{p}$ in the massive case. That is, the signal correlation between the detectors becomes stronger than the massless case at a fixed frequency. At high frequencies, as the phase velocity approaches the speed of light more and more, the overlap reduction functions finally coincide with those in the massless case. As a result, the overlap reduction functions have frequency dependence as if they have shrunk in frequency, particularly at low frequencies. Interestingly, in the massive case, the detectors are much more sensitive to a GWB at slightly higher frequencies than $f_{g}$, compared with the massless case.

\section{SENSITIVITY TO GRAVITON MASS}

\section{A. Fisher matrix}

To estimate the measurement accuracy of graviton mass from the detection of a stochastic GWB, we use the Fisher information matrix derived in Appendix A:

$$
\begin{aligned}
F_{a b}(\vec{\theta})= & C_{0} T \sum_{i=1}^{N_{\mathrm{pair}}} \int_{0}^{\infty} \frac{\Gamma_{a b, i}(f ; \vec{\theta})}{\mathcal{N}_{i}(f) f^{6}} d f, \\
C_{0} \equiv & 2\left(\frac{3 H_{0}^{2}}{10 \pi^{2}}\right)^{2}, \\
\Gamma_{a b, i}(f ; \vec{\theta}) \equiv & \sum_{A, A^{\prime}}\left[\gamma_{i}^{A} \gamma_{i}^{A^{\prime}}\left(\partial_{a} \Omega_{\mathrm{gw}}^{A}\right)\left(\partial_{b} \Omega_{\mathrm{gW}}^{A^{\prime}}\right)\right. \\
& +\left(\partial_{a} \gamma_{i}^{A}\right)\left(\partial_{b} \gamma_{i}^{A^{\prime}}\right) \Omega_{\mathrm{gw}}^{A} \Omega_{\mathrm{gw}}^{A^{\prime}} \\
& +\gamma_{i}^{A}\left(\partial_{b} \gamma_{i}^{A^{\prime}}\right) \Omega_{\mathrm{gw}}^{A^{\prime}}\left(\partial_{a} \Omega_{\mathrm{gw}}^{A}\right) \\
& \left.+\gamma_{i}^{A^{\prime}}\left(\partial_{a} \gamma_{i}^{A}\right) \Omega_{\mathrm{gw}}^{A}\left(\partial_{b} \Omega_{\mathrm{gw}}^{A^{\prime}}\right)\right] .
\end{aligned}
$$

Here, $\partial_{a}$ is the derivative with respect to $\theta_{a}$, and the product of the noise spectra of the $I$ th and $J$ th detectors is $\mathcal{N}_{i}(f)=P_{I}(f) P_{J}(f)$, which is defined for the $i=I J$ detector pair. In our model of the GWB spectrum given in Eq. (21), each polarization is independent, and we have in total nine model parameters: $\vec{\theta}=\left\{\Omega_{\mathrm{gw}, 0}^{A}, n_{t}^{A}, m_{g}^{A}\right\}$ for $A=$ $T, V, S$. Note that the free parameters $m_{g}^{A}$ are included not only in $\Omega_{\mathrm{gw}}^{A}$ but also in $\gamma_{i}^{A}$ so that we have four terms in Eq. (43). Given the numerically evaluated Fisher matrix, the marginalized $1-\sigma$ error of a parameter $\Delta \theta_{a}$ is estimated from the inverse Fisher matrix

$$
\Delta \theta_{a}=\sqrt{\left\{\mathbf{F}^{-1}\right\}_{a a}} .
$$

It is well known that parameter estimation with the Fisher matrix is valid only when the SNR is high enough, e.g., $\gtrsim 10$ [56,57]. For a low SNR, the parameter 
estimation is too optimistic. Therefore, as explained later, we require at least a $\mathrm{SNR}=10$ to estimate the measurement accuracy of the parameters.

Assuming that only a single polarization exists and explicitly assigning the components of the Fisher matrix, the above expression of $F_{a b}$ is much simplified. In the presence of multiple polarization modes, adopting the method proposed in Refs. [19,20], we can separate the mixture of polarization signals and obtain the correlation signal with a single polarization mode. Thus, in this section we assume that only a single polarization is present and omit the superscripts $A$ and $A^{\prime}$ in the expressions below in this section. The parameter estimation in the presence of multiple polarization modes will be discussed in a later section. Depending on whether or not the Fisher matrix contains the derivative with respect to $m_{g}$, it is classified into three cases:

(i) $\theta_{a, b}=\left\{\Omega_{\mathrm{gw}, 0}, n_{t}\right\}$,

(ii) $\theta_{a}=\left\{\Omega_{\mathrm{gw}, 0}, n_{t}\right\}$ and $\theta_{b}=m_{g}$,

(iii) $\theta_{a, b}=m_{g}$.

For $\theta_{a, b}=\left\{\Omega_{\mathrm{gw}, 0}, n_{t}\right\}$, since $\gamma_{i}$ contains only the parameter $m_{g}$, only the first term in Eq. (43) remains:

$F_{a b}=C_{0} T \sum_{i=1}^{N_{\text {pair }}} \int_{0}^{\infty} \frac{\gamma_{i}^{2}\left(\partial_{a} \Omega_{\mathrm{gw}}\right)\left(\partial_{b} \Omega_{\mathrm{gw}}\right)}{\mathcal{N}_{i}(f) f^{6}} d f$ for

$\theta_{a, b}=\left\{\Omega_{\mathrm{gw}, 0}, n_{t}\right\}$.

For $\theta_{a}=\left\{\Omega_{\mathrm{gw}, 0}, n_{t}\right\}$ and $\theta_{b}=m_{g}$, the second and fourth terms in Eq. (43) vanish,

$$
\begin{aligned}
\Gamma_{a m, i}(f ; \vec{\theta})= & \gamma_{i}^{2}\left(\partial_{a} \Omega_{\mathrm{gw}}\right)\left(\partial_{m} \Omega_{\mathrm{gw}}\right) \\
& +\gamma_{i} \Omega_{\mathrm{gw}}\left(\partial_{m} \gamma_{i}\right)\left(\partial_{a} \Omega_{\mathrm{gw}}\right),
\end{aligned}
$$

where $\partial_{m}$ denotes the derivative with respect to $m_{g}$. Using

$$
\partial_{m} \Omega_{\mathrm{gw}}(f)=-\frac{1}{2 \pi} \Omega_{\mathrm{gw}, 0}\left(\frac{f}{f_{0}}\right)^{n_{t}} \delta\left[f-f_{g}\right],
$$

and performing the frequency integral in Eq. (42), we have

$$
\begin{aligned}
F_{a m}= & C_{0} T \sum_{i=1}^{N_{\mathrm{pair}}}\left[\int_{0}^{\infty} \frac{\gamma_{i} \Omega_{\mathrm{gw}}\left(\partial_{m} \gamma_{i}\right)\left(\partial_{a} \Omega_{\mathrm{gw}}\right)}{\mathcal{N}_{i} f^{6}} d f\right. \\
& \left.-\left.\frac{1}{2 \pi}\left\{\Omega_{\mathrm{gw}, 0}\left(\frac{f}{f_{0}}\right)^{n_{t}} \frac{\gamma_{i}^{2}\left(\partial_{a} \Omega_{\mathrm{gw}}\right)}{\mathcal{N}_{i} f^{6}}\right\}\right|_{f=f_{g}}\right], \quad \text { for } \\
\theta_{a}= & \left\{\Omega_{\mathrm{gw}, 0}, n_{t}\right\} \quad \text { and } \quad \theta_{b}=m_{g}
\end{aligned}
$$

For $\theta_{a}, \theta_{b}=m_{g}$, substituting Eq. (47) for Eq. (42) and again performing the frequency integral give

$$
\begin{aligned}
F_{m m}= & C_{0} T \sum_{i=1}^{N_{\mathrm{pair}}}\left[\int_{0}^{\infty} \frac{\left(\partial_{m} \gamma_{i}\right)^{2} \Omega_{\mathrm{gw}}^{2}}{\mathcal{N}_{i} f^{6}} d f\right. \\
& -\left.\frac{1}{\pi}\left\{\Omega_{\mathrm{gw}, 0}^{2}\left(\frac{f}{f_{0}}\right)^{2 n_{t}} \frac{\gamma_{i}\left(\partial_{m} \gamma_{i}\right)}{\mathcal{N}_{i} f^{6}}\right\}\right|_{f=f_{g}} \\
& \left.+\left.\frac{1}{4 \pi^{2}}\left\{\Omega_{\mathrm{gw}, 0}^{2}\left(\frac{f}{f_{0}}\right)^{2 n_{t}} \frac{\gamma_{i}^{2}}{\mathcal{N}_{i} f^{6}} \delta\left[f-f_{g}\right]\right\}\right|_{f=f_{g}}\right]
\end{aligned}
$$

In the third term of the above equation, $\delta(0)$ appears since the delta function has a support exactly at $f=f_{g}$ and otherwise zero. However, in a real detector we cannot exactly measure the frequency $f_{g}$ and then we ignore the third term.

To compute the Fisher matrix, what we need is the expressions for $\partial_{a} \Omega_{\mathrm{gw}}$ and $\partial_{m} \gamma_{i}$. From Eq. (21),

$$
\begin{gathered}
\frac{\partial \Omega_{\mathrm{gw}}(f)}{\partial \Omega_{\mathrm{gw}, 0}}=\left(\frac{f}{f_{0}}\right)^{n_{t}} \Theta\left[f-f_{g}\right], \\
\frac{\partial \Omega_{\mathrm{gw}}(f)}{\partial n_{t}}=\Omega_{\mathrm{gw}}(f) \log \left[\frac{f}{f_{0}}\right] .
\end{gathered}
$$

As for $\partial_{m} \gamma_{i}$, first let us write the overlap reduction functions in Eqs. (33)-(41) in a compact form as

$$
\begin{aligned}
& \gamma^{A}\left(\alpha, \beta, \sigma_{+}, \sigma_{-}\right) \\
& \quad=\Theta_{A+}(\alpha, \beta) \cos \left(4 \sigma_{+}\right)+\Theta_{A_{-}}(\alpha, \beta) \cos \left(4 \sigma_{-}\right),
\end{aligned}
$$

with

$$
\begin{aligned}
& \Theta_{A^{+}}(\alpha, \beta)=a_{0}^{A}(\beta) j_{0}+a_{2}^{A}(\beta) j_{2}+a_{4}^{A}(\beta) j_{4}, \\
& \Theta_{A^{-}}(\alpha, \beta)=b_{0}^{A}(\beta) j_{0}+b_{2}^{A}(\beta) j_{2}+b_{4}^{A}(\beta) j_{4} .
\end{aligned}
$$

Differentiating Eq. (52) with respect to $m_{g}^{A}$ and using the recursion relation of the spherical Bessel functions, we have

$$
\begin{aligned}
\partial_{m} \gamma^{A}= & -\frac{\sqrt{v_{p}^{2}-1}}{\omega_{c}}\left[\left(\partial_{\alpha} \Theta_{A+}\right) \cos \left(4 \sigma_{+}\right)+\left(\partial_{\alpha} \Theta_{A-}\right)\right. \\
& \left.\times \cos \left(4 \sigma_{-}\right)\right] \\
\partial_{\alpha} \Theta_{A+}= & \left(-a_{0}+\frac{2}{5} a_{2}\right) j_{1}+\left(-\frac{3}{5} a_{2}+\frac{4}{9} a_{4}\right) j_{3}-\frac{5}{9} a_{4} j_{5}, \\
\partial_{\alpha} \Theta_{A-}= & \left(-b_{0}+\frac{2}{5} b_{2}\right) j_{1}+\left(-\frac{3}{5} b_{2}+\frac{4}{9} b_{4}\right) j_{3}-\frac{5}{9} b_{4} j_{5}
\end{aligned}
$$

where the coefficients $a_{i}, b_{i}(i=0,2,4)$ are functions of $\beta$ and different for each polarization mode, and $\omega_{c} \equiv c /|\Delta \mathbf{X}|$. 

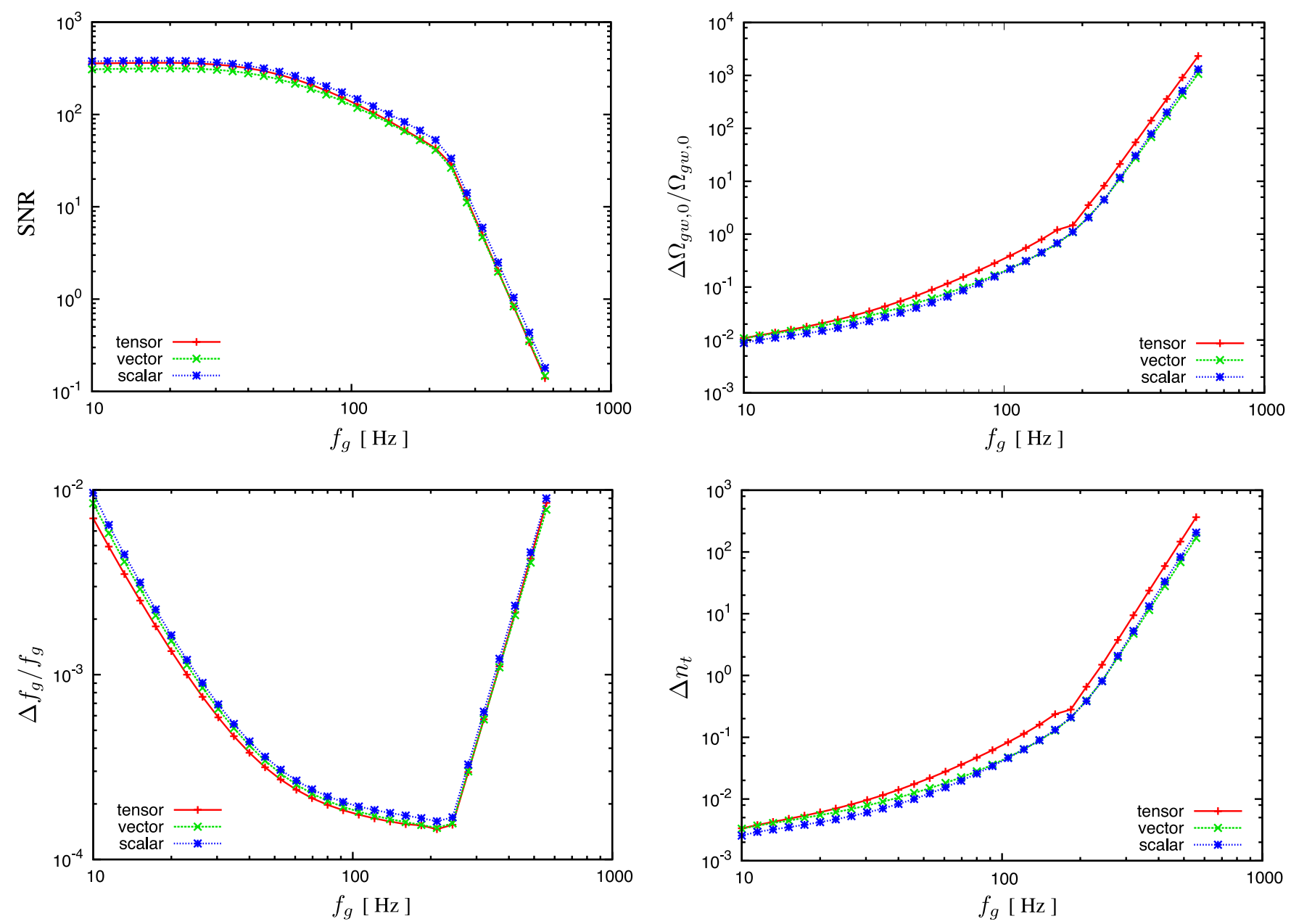

FIG. 3 (color online). SNR and the parameter estimation accuracy as a function of a fiducial value of $f_{g}$ in $\mathrm{Hz}$ in the presence of a single polarization mode. The fiducial values of the other parameters are $h_{0}^{2} \Omega_{\mathrm{gw}, 0}=10^{-7}$ and $n_{t}=0$. The top left is the SNR, the top right is $\Delta \Omega_{\mathrm{gw}, 0} / \Omega_{\mathrm{gw}, 0}$, the bottom left is $\Delta f_{g} / f_{g}$, and the bottom right is $\Delta n_{t}$. The red, green, and blue curves are the tensor, vector, and scalar modes, respectively.

\section{B. Signal-to-noise ratio}

The parameter estimation with the Fisher information is valid in a high-SNR regime. If the SNR is low, e.g., SNR $<10$, the measurement accuracy of the parameters could be overestimated. To make the results derived with the Fisher matrix reliable, we will first check the SNRs for a stochastic GWB.
Using the SNR formula in Eq. (30), we evaluate the SNR for each polarization mode with a detector pair, assuming that only one polarization mode (tensor, vector, or scalar mode) exists and a 3-yr observation time. As for the power spectra of the detector noise $P_{I}(f)$, we use for simplicity that of aLIGO for all advanced detectors $(\mathrm{K}, \mathrm{H}, \mathrm{L}, \mathrm{V})$, which is based on Ref. [58] and given by Ref. [41]

$$
P(f)= \begin{cases}10^{-44}\left(\frac{f}{10 \mathrm{~Hz}}\right)^{-4}+10^{-47.25}\left(\frac{f}{100 \mathrm{~Hz}}\right)^{-1.7} \mathrm{~Hz}^{-1} & \text { for } 10 \mathrm{~Hz} \leq f \leq 240 \mathrm{~Hz} \\ 10^{-46}\left(\frac{f}{1000 \mathrm{~Hz}}\right)^{3} \mathrm{~Hz}^{-1} & \text { for } 240 \mathrm{~Hz} \leq f \leq 3000 \mathrm{~Hz} \\ \infty & \text { otherwise. }\end{cases}
$$

The lower and higher cutoffs of the frequency integral are set to $f_{\text {low }}=10 \mathrm{~Hz}$ and $f_{\text {high }}=1 \mathrm{kHz}$.

For a detector network, $(\mathrm{SNR})^{2}$ is given by the squared sum of SNR of each detector pair,

$$
(\mathrm{SNR})^{2}=\sum_{i=1}^{N_{\text {pair }}}\left(\mathrm{SNR}_{i}\right)^{2},
$$


where $N_{\text {pair }}$ is the number of detector pairs. In Fig. 3, we show the SNR of a detector network $(\mathrm{K}, \mathrm{H}, \mathrm{L}, \mathrm{V})$ for a stochastic GWB of $h_{0}^{2} \Omega_{\mathrm{gw}, 0}^{A}=10^{-7}$ and $n_{t}^{A}=0$ as a function of graviton-mass frequency $f_{g}^{A}$. At low $f_{g}$, since the GWB spectrum is indistinguishable from the massless case, the SNR coincides with that in the massless case. As $f_{g}$ increases, the SNR gradually decreases due to the low-frequency cutoff at $f=f_{g}$ of the GWB spectrum. For the fiducial parameters $h_{0}^{2} \Omega_{\mathrm{gw}, 0}^{A}=10^{-7}$ and $n_{t}^{A}=0$, if we require $\mathrm{SNR}=10$ for reliable detection, this implies that massive GWB with $f_{g}$ less than $\sim 300 \mathrm{~Hz}$ is detectable. As is clear from the SNR formula in Eq. (30), the SNR scales linearly with $\Omega_{\mathrm{gw}, 0}$. For $h_{0}^{2} \Omega_{\mathrm{gw}, 0}^{A}=10^{-8}$, the SNR is

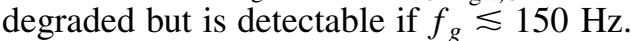

\section{Sensitivity to graviton mass}

We calculate the Fisher matrix with free parameters, $\left\{\Omega_{\mathrm{gw}, 0}^{A}, n_{t}^{A}, m_{g}^{A}\right\}, A=T, V, S$, assuming that only a single polarization mode exists (the superscript $A$ is omitted below). The parameter estimation accuracy with a detector network is computed by adding the Fisher matrices of all detector pairs. The pivot frequency $f_{0}$ of the spectral index $n_{t}$ is chosen as $f_{0}=1 \mathrm{~Hz}$, which is outside of the observation frequency band in order to avoid insensitivity to $n_{t}$ at $f=f_{0}$. The fiducial parameters are set to $h_{0}^{2} \Omega_{\mathrm{gw}, 0}=$ $10^{-7}$ and $n_{t}=0$.

The results are shown in Fig. 3 as a function of the fiducial values of $f_{g}$. The measurement errors of $\Omega_{\mathrm{gw}, 0}$ and $n_{t}$ are smaller at low $f_{g}$ and approach those in the massless case at the limit of $f_{g} \rightarrow 0$. This is because the contribution to the Fisher matrix is an integrated quantity in frequency. As $f_{g}$ increases, the measurement accuracies of $\Omega_{\mathrm{gw}, 0}$ and $n_{t}$ are deteriorated. At $f_{g}$ slightly below the high-frequency cutoff required by the SNR threshold 10 , i.e., $\sim 200 \mathrm{~Hz}$, the parameters $\Omega_{\mathrm{gw}, 0}$ and $n_{t}$ are still measurable. On the other hand, for the measurement accuracy of $m_{g}$ or $f_{g}$, the detector network is most sensitive to the value of $f_{g}$ around the minimum of the detector noise curve, $\sim 100 \mathrm{~Hz}$. This means that the dominant contribution to the Fisher matrix comes from the frequencies around $f \sim f_{g}$. Interestingly, the parameter $f_{g}$ is well determined for all $f_{g}$ in the frequency band of a ground-based detector.

From the above results, we conclude that graviton mass is detectable for a massive GWB of $h_{0}^{2} \Omega_{\mathrm{gw}, 0}^{A}=10^{-7}$ and $n_{t}^{A}=0$ if the graviton mass is in the range

$$
\begin{gathered}
6.6 \times 10^{-15} \mathrm{eV} \leq m_{g}^{A} \leq 1.8 \times 10^{-13} \mathrm{eV}, \\
\text { for } A=T, V, S .
\end{gathered}
$$

When the amplitude of a GWB is different from $h_{0}^{2} \Omega_{\mathrm{gw}, 0}^{A}=$ $10^{-7}$, the Fisher matrix just scales with the square of the amplitude. Thus, the measurement accuracy of $f_{g}$ inversely scales with the amplitude, namely, $\Delta f_{g} / f_{g} \propto$ $\left(\Omega_{\mathrm{gw}, 0}\right)^{-1}$. We also have another criterion for mass detection, SNR $>10$, which guarantees reliable parameter estimation based on the Fisher matrix. As seen in Fig. 3, the SNR criterion is tighter in our calculation. Then, we obtain the detectable parameter region of $m_{g}$ and $\Omega_{\mathrm{gw}, 0}$ when $n_{t}^{A}=0$, which is shown in Fig. 4. Below the curves, the GWB is not detected with a sufficient SNR and graviton mass cannot be determined. The slight differences of the detection threshold of graviton mass are due to the small differences of the antenna pattern function of each polarization mode.

One might wonder how the detectable parameter region of graviton mass is changed if the fiducial value of $n_{t}$ deviates from zero. Since our choice of the pivot frequency of the GWB spectrum is $f_{0}=1 \mathrm{~Hz}$, the blue (red) tilt of the spectrum just increases (decreases) the SNR more at high frequencies. Then, positive (negative) $n_{t}$ expands (reduces) the detectable parameter region of graviton mass in Fig. 4.

Finally, we remark that we can set an upper limit on graviton mass if a GWB is detected but the lower frequency cutoff is not detected. In this case, the lower frequency cutoff of the GWB spectrum is at least below the frequency band of a detector. Therefore, we find the mass limit $f_{g}<f_{\text {low }}$, where $f_{\text {low }}$ is the lower cutoff of the detector noise curve and say, $\sim 10 \mathrm{~Hz}$. In this case, the mass limit will be $m_{g}<6.6 \times 10^{-15} \mathrm{eV}$. For the tensor mode, this mass constraint is much weaker than what was obtained from the observation of binary pulsars, $m_{g}<7.6 \times$ $10^{-20} \mathrm{eV}$ [46]. However, it is worthy to note that the bounds on graviton mass are obtained from different observations of GWs at completely different environments at binary pulsars and on Earth, because it is possible to give gravitons larger mass on Earth if an environment-dependent screening effect such as the chameleon mechanism would occur.

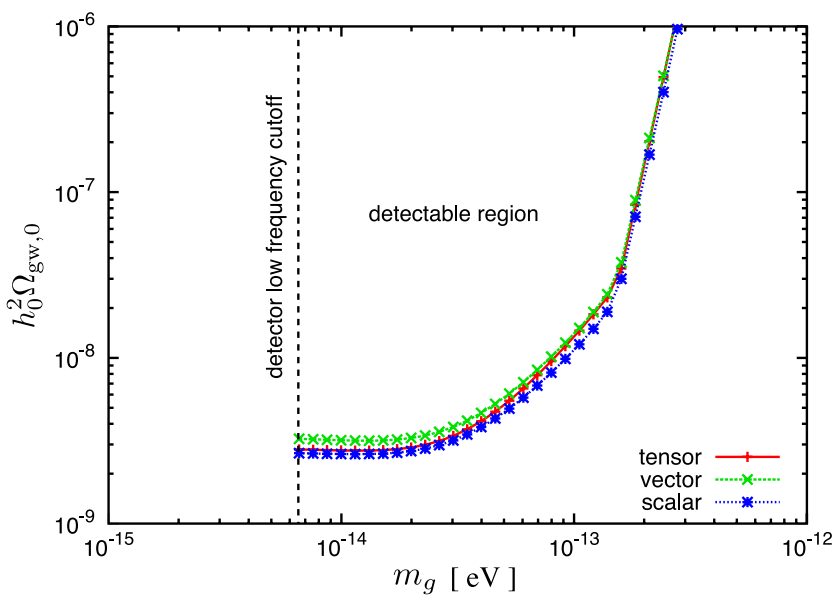

FIG. 4 (color online). Detectable parameter region of $m_{g}$ and $\Omega_{\mathrm{gw}, 0}$ when $n_{t}^{A}=0$. The red, green, and blue curves are the detection thresholds for the tensor, vector, and scalar modes, respectively. The region above the curves is detectable with a detector network. 


\section{MIXTURE OF POLARIZATION MODES AND ITS SEPARABILITY}

In the previous section, we computed the parameter estimation accuracy of the GWB spectrum, particularly focusing on the measurement accuracy of the graviton mass, in the presence of a single polarization mode. However, if there exist massive polarization modes due to the modification of gravity theory, what we observe is likely to be a mixture signal of different polarizations. For example, if a gravity theory has an additional scalar degree of freedom, a GWB would be composed of an ordinary massless tensor mode and massive scalar mode. To this end, in this section we investigate the parameter estimation in the presence of a mixed GWB. We assume that a tensor graviton is massless, $m_{g}^{T}=0$ [59] since graviton mass in a tensor mode has already been tightly constrained, as mentioned in Sec. II D. Also, for simplicity we consider the case of flat GWB spectra, $n_{t}^{A}=0, A=T, V, S$. With these fiducial values, we estimate the measurement accuracy of five parameters: $\Omega_{\mathrm{gw}, 0}^{A}(A=T, V, S), m_{g}^{V}, m_{g}^{S}$.

To do so, we need extra terms of the Fisher matrix arising from cross correlation between different polarization modes, in addition to the autocorrelation terms provided in Sec. IVA. The explicit forms of the elements of the Fisher matrix are obtained in the same way as in Sec. IVA and are given in Appendix B.

First, we study the effect of mass degeneracy. The fiducial values of the spectral amplitude are set to $h_{0}^{2} \Omega_{\mathrm{gw}, 0}^{A}=10^{-7}$ for all polarization modes. The vector and scalar graviton masses are set to different values. The result is shown in Fig. 5. When the vector and scalar graviton masses are equal, there is weak degeneracy of the parameters, which slightly worsens the measurement accuracies of $f_{g}^{V}$ and $f_{g}^{S}$. If the vector graviton mass is set to $f_{g}^{V}=40 \mathrm{~Hz}$, the measurement accuracy of $f_{g}^{S}$ is improved because of the broken degeneracy of the parameters and is almost the same as the one we obtained in the previous section in the presence of a single scalar polarization mode. This indicates that in general the mixture of polarization signals can be well separated by data-analysis procedures unless the parameters are degenerated. Such a method has been already proposed and investigated in Refs. [19,20] in the case of the massless GWB. The authors have shown that at least three correlation signals allow one to separate three polarization modes, without any degeneracy between polarizations in the case of advanced ground-based detectors that we consider in this paper, so the good separability of the polarization modes is manifest even if the graviton has its mass.

Second, we investigate how different amplitudes between the polarization modes affect the measurement accuracy of graviton mass. In Fig. 6, the result is shown. If a GWB spectrum is the sum of the large tensor mode with $h_{0}^{2} \Omega_{\mathrm{gw}, 0}^{T}=10^{-7}$ and small vector and scalar modes with $h_{0}^{2} \Omega_{\mathrm{gw}, 0}^{V, S}=10^{-8}$, the measurement accuracies of $f_{g}^{V}$ and $f_{g}^{S}$

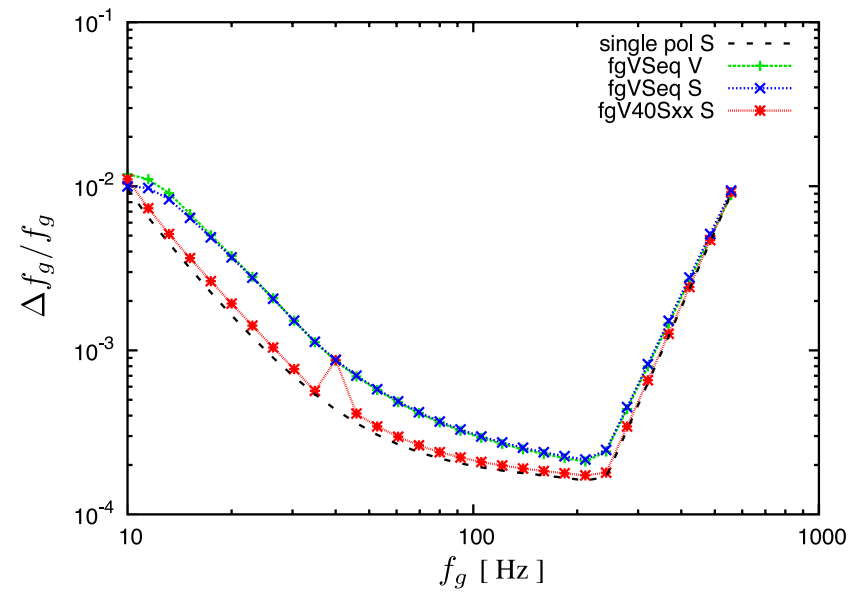

FIG. 5 (color online). Measurement accuracy of graviton mass when all polarization signals are mixed and each mode has different graviton mass. In the legend, fgVSeq V and fgVSeq $\mathrm{S}$ are the measurement accuracies of $f_{g}^{V}$ and $f_{g}^{S}$ when both have equal masses $f_{g}=f_{g}^{V}=f_{g}^{S}$. While fgV40Sxx S is the measurement accuracy of $f_{g}^{S}$ when the vector graviton mass is fixed to $f_{g}^{V}=40 \mathrm{~Hz}$ and $f_{g}=f_{g}^{S}$. The dashed curve is the one we obtained in the previous section in the presence of a scalar polarization mode. In all cases, the fiducial amplitude is $h_{0}^{2} \Omega_{\mathrm{gw}, 0}^{A}=10^{-7}$ for all polarization modes.

just inversely scale with the power amplitude. Again, we can conclude that the mixture of polarization signals can be well separated by a data-analysis procedure even if the GWB spectrum of each polarization mode has different amplitude.

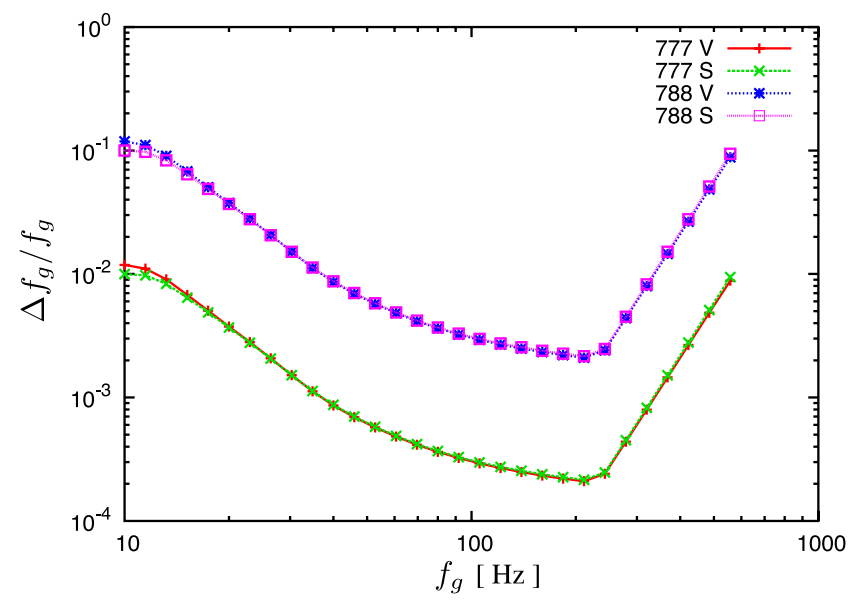

FIG. 6 (color online). Measurement accuracy of graviton mass when all polarization signals are mixed and each mode has a different GWB amplitude. Vector and scalar graviton masses are set to $f_{g}=f_{g}^{V}=f_{g}^{S}$. In the legend, $777 \mathrm{~V}$ and $777 \mathrm{~S}$ represent the measurement accuracies of $f_{g}^{V}$ and $f_{g}^{S}$ when the fiducial amplitude is $h_{0}^{2} \Omega_{\mathrm{gw}, 0}^{A}=10^{-7}$ for all polarization modes; $788 \mathrm{~V}$ and $788 \mathrm{~S}$ are those when the fiducial amplitudes are $h_{0}^{2} \Omega_{\mathrm{gw}, 0}^{T}=$ $10^{-7}$ and $h_{0}^{2} \Omega_{\mathrm{gw}, 0}^{V}=h_{0}^{2} \Omega_{\mathrm{gw}, 0}^{S}=10^{-8}$. 


\section{CONCLUSIONS}

We studied the search method for a massive stochastic GWB, extending the ordinary method for massless graviton to that for massive graviton and allowing the tensor-, vector-, and scalar-polarization modes of the GWB. If a GWB is massive, the phase velocity, which is faster than the speed of light, affects the cross-correlation statistics by changing the effective distance between the detectors. Also, there is a lower frequency cutoff on a GWB spectrum that corresponds to graviton mass.

To investigate the detectability of graviton mass with ground-based detectors, we used a generically parametrized model of a GWB spectrum and estimated the measurement accuracies of the parameters with the Fisher information matrix. As a result, we found that if a GWB is detected at the level of $h_{0}^{2} \Omega_{\mathrm{gw}, 0}=10^{-7}$, we could determine the mass of graviton in the range of $7 \times$ $10^{-15} \mathrm{eV} \lesssim m_{g} \lesssim 2 \times 10^{-13} \mathrm{eV}$ for each polarization mode with ground-based detectors. We also showed that even if the GWB signal is a mixture of three polarization modes, we could safely separate them and determine the mass of the graviton. Even if a GWB is detected but the lower frequency cutoff is not detected, we can set an upper limit on graviton mass, which is the lower cutoff of the detector noise curve, say, $\sim 10 \mathrm{~Hz}$ or $\sim 7 \times 10^{-15} \mathrm{eV}$.

Finally, note that our method to search for graviton mass can be a quite generic and model-independent test of an alternative theory of gravity because we did not assume any specific model of gravity theory in the reconstruction of a GWB spectral shape. The constraints on the polarization degrees of freedom, graviton mass, spectral index, and spectral amplitude would help select out or exclude some specific models of gravity. In this paper, although we focused on ground-based detectors such as aLIGO, aVIRGO, and KAGRA, it would be interesting to consider the constraints obtained in the future with other detectors such as ET [60], LISA [61], DECIGO [62], BBO [63], and pulsar timing array [64], since different observational frequency bands correspond to different mass ranges of a graviton. In particular, among GW detectors, LISA is sensitive in the lowest frequency band as low as $\sim 10^{-4} \mathrm{~Hz}$, which is roughly 5-6 orders lower than the frequency band of a ground-based detector. If a GWB is detected by LISA but the low-frequency cutoff of the spectrum is not detected, the graviton mass constraint for the tensor mode would be comparable to the binary pulsar constraint less than $\sim 10^{-20} \mathrm{eV}$.

\section{ACKNOWLEDGMENTS}

We are much obliged to an anonymous referee for his/ her valuable comments to improve the manuscript. A. N. would like to thank N. Seto for helpful discussions. K. H. would like to thank Bruce Allen for warm hospitality during his stay in Hannover and Soumya D. Mohanty for valuable comments and encouragement. A. N. and K. H. are supported by a Grant-in-Aid for Scientific Research on Innovative Areas, Grant No. 24103006.

\section{APPENDIX A: DERIVATION OF FISHER MATRIX}

Here we will derive the Fisher matrix for estimating the measurement accuracy of the parameters of a GWB. When one correlates the detector signals in a frequency bin $\Delta f$, the estimated value of the correlation signal of the $i$ th detector pair (for $I$ th and $J$ th detector pair, $i=I J) \hat{\mu}_{i}(f)$ fluctuates around the true value, $\mu_{i}(f ; \vec{\theta})$, where $\vec{\theta}$ is a set of estimated parameters. Assuming the width of a single frequency bin that we analyze is much larger than the frequency resolution of the data but small enough to neglect the frequency dependence of physical quantity, a likelihood function for $\hat{\mu}_{i}(f)$ is expected to be a Gaussian distribution, owing to the central limit theorem. Then the multidimensional likelihood function for $N_{\text {pair }}$ detector pairs is written as

$$
L\left[\left\{\hat{\mu}_{i}(f)\right\}\right] \propto \exp \left[-\sum_{i=1}^{N_{\text {pair }}} \frac{\left\{\hat{\mu}_{i}(f)-\mu_{i}(f ; \vec{\theta})\right\}^{2}}{2 \sigma_{i}^{2}(f)}\right] .
$$

Suppose that $\vec{\theta}_{M L}$ are the maximum likelihood values of the parameters and denote the small deviation from it by $\Delta \vec{\theta}$. The likelihood function can be expanded around its peak $\vec{\theta}_{M L}$ and by neglecting the first derivative it reduced to

$L\left[\left\{\hat{\mu}_{i}(f)\right\}\right] \propto \exp \left[-\sum_{i=1}^{N_{\text {pair }}} \sum_{a, b} \frac{\partial_{a} \mu_{i}(f ; \vec{\theta}) \partial_{b} \mu_{i}(f ; \vec{\theta})}{2 \sigma_{i}^{2}(f)}\right]$.

From this expression, the Fisher matrix reads

$$
F_{a b}(f)=\sum_{i=1}^{N_{\text {pair }}} \frac{\partial_{a} \mu_{i}(f ; \vec{\theta}) \partial_{b} \mu_{i}(f ; \vec{\theta})}{2 \sigma_{i}^{2}(f)} .
$$

The correlation signal and noise variation are given in Eqs. (23) and (27) as

$$
\begin{gathered}
\mu_{i}(f ; \vec{\theta})=\frac{3 H_{0}^{2}}{10 \pi^{2}} T f^{-3} \sum_{A} \gamma_{i}^{A}(f ; \vec{\theta}) \Omega_{\mathrm{gw}}^{A}(f ; \vec{\theta}) \Delta f, \\
\sigma^{2}(f)=\frac{T}{2} \mathcal{N}_{i}(f) \Delta f
\end{gathered}
$$

where we defined $\mathcal{N}_{i}(f) \equiv P_{I}(f) P_{J}(f)$. Note that these quantities are defined in a positive frequency range, $0<$ $f<\infty$, and a factor of 2 differs from Eqs. (23) and (27). Substituting Eqs. (A4) and (A5) into Eq. (A3) and integrating over frequency, we obtain

$$
F_{a b}=\sum_{i=1}^{N_{\text {pair }}} 2\left(\frac{3 H_{0}^{2}}{10 \pi^{2}}\right)^{2} T \int_{0}^{\infty} \frac{\Gamma_{a b}(f ; \vec{\theta})}{\mathcal{N}_{i}(f) f^{6}} d f,
$$




$$
\begin{aligned}
\Gamma_{a b}(f ; \vec{\theta}) \equiv & \sum_{A, A^{\prime}}\left[\gamma_{i}^{A} \gamma_{i}^{A^{\prime}}\left(\partial_{a} \Omega_{\mathrm{gW}}^{A}\right)\left(\partial_{b} \Omega_{\mathrm{gW}}^{A^{\prime}}\right)\right. \\
& +\left(\partial_{a} \gamma_{i}^{A}\right)\left(\partial_{b} \gamma_{i}^{A^{\prime}}\right) \Omega_{\mathrm{gw}}^{A} \Omega_{\mathrm{gW}}^{A^{\prime}} \\
& +\gamma_{i}^{A}\left(\partial_{b} \gamma_{i}^{A^{\prime}}\right) \Omega_{\mathrm{gW}}^{A^{\prime}}\left(\partial_{a} \Omega_{\mathrm{gW}}^{A}\right) \\
& \left.+\gamma_{i}^{A^{\prime}}\left(\partial_{a} \gamma_{i}^{A}\right) \Omega_{\mathrm{gW}}^{A}\left(\partial_{b} \Omega_{\mathrm{gW}}^{A^{\prime}}\right)\right] .
\end{aligned}
$$

Here $\partial_{a}$ is the derivative with respect to $\theta_{a}$.

\section{APPENDIX B: CROSS-CORRELATED TERMS OF FISHER MATRIX BETWEEN DIFFERENT POLARIZATIONS}

To calculate the measurement accuracy of graviton mass for a mixture signal of GWBs in different polarization modes, we need extra terms of the Fisher matrix arising from cross correlation between different polarizations, in addition to the autocorrelation terms provided in Sec. IVA. Here we write down explicit forms of the cross-correlated terms between different polarizations. The extra terms are classified into three cases:

(i) $\theta_{a}=\Omega_{\mathrm{gw}, 0}^{A}, \theta_{b}=\Omega_{\mathrm{gw}, 0}^{A^{\prime}}, A \neq A^{\prime}$,

(ii) $\theta_{a}=\Omega_{\mathrm{gw}, 0}^{A}, \theta_{b}=m_{g}^{A^{\prime}}, A \neq A^{\prime}$,

(iii) $\theta_{a}=m_{g}^{A}, \theta_{b}=m_{g}^{A^{\prime}}, A \neq A^{\prime}$.

Case (i) has the additional term

$$
\begin{aligned}
\Gamma_{\Omega \Omega, i}^{A A^{\prime}}(f ; \vec{\theta}) & =\gamma_{i}^{A} \gamma_{i}^{A^{\prime}}\left(\partial_{a} \Omega_{\mathrm{gw}}^{A}\right)\left(\partial_{b} \Omega_{\mathrm{gw}}^{A^{\prime}}\right) \\
& =\gamma_{i}^{A} \gamma_{i}^{A^{\prime}} \Theta\left[f-f_{g}^{A}\right] \Theta\left[f-f_{g}^{A^{\prime}}\right] \\
& =\gamma_{i}^{A} \gamma_{i}^{A^{\prime}} \Theta\left[f-\max \left(f_{g}^{A}, f_{g}^{A^{\prime}}\right)\right] .
\end{aligned}
$$

The frequency integral in Eq. (42) is performed above the frequency $\max \left(f_{g}^{A}, f_{g}^{A^{\prime}}\right)$. We have

$$
F_{\Omega \Omega}^{A A^{\prime}}=C_{0} T \sum_{i=1}^{N_{\text {pair }}} \int_{\max \left(f_{g}^{A}, f_{g}^{A^{\prime}}\right)}^{\infty} \frac{\gamma_{i}^{A} \gamma_{i}^{A^{\prime}}}{\mathcal{N}_{i}(f) f^{6}} d f
$$

Case (ii) has the additional term

$$
\begin{aligned}
\Gamma_{\Omega m, i}^{A A^{\prime}}(f ; \vec{\theta})= & -\frac{1}{2 \pi} \Omega_{\mathrm{gw}, 0}^{A^{\prime}} \gamma_{i}^{A} \gamma_{i}^{A^{\prime}} \Theta\left[f-f_{g}^{A}\right] \delta\left[f-f_{g}^{A^{\prime}}\right] \\
& +\gamma_{i}^{A} \Omega_{\mathrm{gw}, 0}^{A^{\prime}}\left(\partial_{m} \gamma_{i}^{A^{\prime}}\right) \Theta\left[f-f_{g}^{A}\right] .
\end{aligned}
$$

After the frequency integration, we have

$$
\begin{aligned}
F_{\Omega m}^{A A^{\prime}}= & C_{0} T \sum_{i=1}^{N_{\mathrm{pair}}}\left[\int_{f_{g}^{A}}^{\infty} \frac{\gamma_{i}^{A} \Omega_{\mathrm{gw}}^{A^{\prime}}\left(\partial_{m} \gamma_{i}^{A^{\prime}}\right)}{\mathcal{N}_{i} f^{6}} d f\right. \\
& \left.-\left.\frac{\Omega_{\mathrm{gw}, 0}^{A^{\prime}}}{2 \pi}\left\{\frac{\gamma_{i}^{A} \gamma_{i}^{A^{\prime}}}{\mathcal{N}_{i} f^{6}}\right\}\right|_{f=f_{g}^{A^{\prime}}} \Theta\left[f_{g}^{A^{\prime}}-f_{g}^{A}\right]\right] .
\end{aligned}
$$

Case (iii) has the additional term

$$
\begin{aligned}
F_{m m}^{A A^{\prime}}= & C_{0} T \Omega_{\mathrm{gw}}^{A} \Omega_{\mathrm{gw}}^{A^{\prime}} \sum_{i=1}^{N_{\mathrm{pair}}}\left[\int_{\max \left(f_{g}^{A}, f_{g}^{A^{\prime}}\right)}^{\infty} \frac{\left(\partial_{m} \gamma_{i}^{A}\right)\left(\partial_{m} \gamma_{i}^{A^{\prime}}\right)}{\mathcal{N}_{i} f^{6}} d f\right. \\
& -\left.\frac{1}{2 \pi}\left\{\frac{\gamma_{i}^{A}\left(\partial_{m} \gamma_{i}^{A^{\prime}}\right)}{\mathcal{N}_{i} f^{6}}\right\}\right|_{f=f_{g}^{A}} \Theta\left[f_{g}^{A}-f_{g}^{A^{\prime}}\right] \\
& \left.-\left.\frac{1}{2 \pi}\left\{\frac{\left(\partial_{m} \gamma_{i}^{A}\right) \gamma_{i}^{A^{\prime}}}{\mathcal{N}_{i} f^{6}}\right\}\right|_{f=f_{g}^{A^{\prime}}} \Theta\left[f_{g}^{A^{\prime}}-f_{g}^{A}\right]\right] .
\end{aligned}
$$

[1] Advanced LIGO webpages https://www .advancedligo.mit.edu/.

[2] Advanced VIRGO webpage https://www.cascina .virgo.infn.it/advirgo/.

[3] KAGRA webpage http://gwcenter.icrr.u-tokyo.ac.jp/en/.

[4] C. V. D. Broeck, arXiv:1301.7291.

[5] J.R. Gair, M. Vallisneri, S.L. Larson, and J.G. Baker, arXiv:1212.5575 [Living Rev. Relativity (to be published)].

[6] N. Yunes and X. Siemens, arXiv:1304.3473 [Living Rev. Relativity (to be published)].

[7] C. Cutler and E. E. Flanagan, Phys. Rev. D 49, 2658 (1994).

[8] C. K. Mishra, K. Arun, B. R. Iyer, and B. Sathyaprakash, Phys. Rev. D 82, 064010 (2010).

[9] W. Del Pozzo, J. Veitch, and A. Vecchio, Phys. Rev. D 83, 082002 (2011).

[10] T. Li, W. Del Pozzo, S. Vitale, C. Van Den Broeck, M. Agathos, J. Veitch, K. Grover, T. Sidery, R. Sturani, and A. Vecchio, Phys. Rev. D 85, 082003 (2012).

[11] N. Yunes and F. Pretorius, Phys. Rev. D 80, 122003 (2009).
[12] N. Yunes and S. A. Hughes, Phys. Rev. D 82, 082002 (2010).

[13] N. Cornish, L. Sampson, N. Yunes, and F. Pretorius, Phys. Rev. D 84, 062003 (2011).

[14] D. M. Eardley, D. L. Lee, A. P. Lightman, R. V. Wagoner, and C. M. Will, Phys. Rev. Lett. 30, 884 (1973).

[15] C. M. Will, Living Rev. Relativity 9, 3 (2006).

[16] M.E. Alves, O.D. Miranda, and J.C. de Araujo, Phys. Lett. B 679, 401 (2009).

[17] M. Alves, O. Miranda, and J. de Araujo, Classical Quantum Gravity 27, 145010 (2010).

[18] C. de Rham, G. Gabadadze, and A. J. Tolley, J. High Energy Phys. 11 (2011) 093.

[19] A. Nishizawa, A. Taruya, K. Hayama, S. Kawamura, and M.-a. Sakagami, Phys. Rev. D 79, 082002 (2009).

[20] A. Nishizawa, A. Taruya, and S. Kawamura, Phys. Rev. D 81, 104043 (2010).

[21] K. Chatziioannou, N. Yunes, and N. Cornish, Phys. Rev. D 86, 022004 (2012).

[22] K. Hayama and A. Nishizawa, Phys. Rev. D 87, 062003 (2013).

[23] C. M. Will, Phys. Rev. D 57, 2061 (1998). 
[24] K. Yagi and T. Tanaka, Phys. Rev. D 81, 064008 (2010).

[25] A. Stavridis and C. M. Will, Phys. Rev. D 80, 044002 (2009).

[26] E. Berti, J. Gair, and A. Sesana, Phys. Rev. D 84, 101501 (2011).

[27] K. Lee, F. A. Jenet, R. H. Price, N. Wex, and M. Kramer, Astrophys. J. 722, 1589 (2010).

[28] M. Maggiore and A. Nicolis, Phys. Rev. D 62, 024004 (2000).

[29] M. Gasperini and C. Ungarelli, Phys. Rev. D 64, 064009 (2001).

[30] K. J. Lee, F. A. Jenet, and R. H. Price, Astrophys. J. 685, 1304 (2008).

[31] T. P. Sotiriou and V. Faraoni, Rev. Mod. Phys. 82, 451 (2010).

[32] A. De Felice and S. Tsujikawa, Living Rev. Relativity 13, 3 (2010).

[33] Y. Fujii and K. Maeda, The Scalar-Tensor Theory of Gravitation (Cambridge University Press, Cambridge, England, 2002).

[34] C.M. Will, Theory and Experiment in Gravitational Physics (Cambridge University Press, Cambridge, England, 1993).

[35] M.E. Tobar, T. Suzuki, and K. Kuroda, Phys. Rev. D 59, 102002 (1999).

[36] B. Allen and J.D. Romano, Phys. Rev. D 59, 102001 (1999).

[37] M. Maggiore, Phys. Rep. 331, 283 (2000).

[38] N. Seto, Phys. Rev. Lett. 97, 151101 (2006).

[39] N. Seto, Phys. Rev. D 75, 061302 (2007).

[40] N. Seto and A. Taruya, Phys. Rev. Lett. 99, 121101 (2007).

[41] N. Seto and A. Taruya, Phys. Rev. D 77, 103001 (2008).

[42] M. S. Turner, M. J. White, and J.E. Lidsey, Phys. Rev. D 48, 4613 (1993).

[43] A. E. Gumrukcuoglu, S. Kuroyanagi, C. Lin, S. Mukohyama, and N. Tanahashi, Classical Quantum Gravity 29, 235026 (2012).

[44] A. S. Goldhaber and M. M. Nieto, Phys. Rev. D 9, 1119 (1974).
[45] C. Talmadge, J.-P. Berthias, R. W. Hellings, and E. M. Standish, Phys. Rev. Lett. 61, 1159 (1988).

[46] L. S. Finn and P. J. Sutton, Phys. Rev. D 65, 044022 (2002).

[47] J. Khoury and A. Weltman, Phys. Rev. D 69, 044026 (2004).

[48] J. Khoury and A. Weltman, Phys. Rev. Lett. 93, 171104 (2004).

[49] B. Bertotti, L. Iess, and P. Tortora, Nature (London) 425, 374 (2003).

[50] A. Vainshtein, Phys. Lett. 39B, 393 (1972).

[51] E. Babichev and C. Deffayet, arXiv:1304.7240.

[52] K. Yagi, A. Nishizawa, and C.-M. Yoo, J. Cosmol. Astropart. Phys. 04 (2012) 031.

[53] S. Camera and A. Nishizawa, Phys. Rev. Lett. 110, 151103 (2013).

[54] N. Christensen, Phys. Rev. D 46, 5250 (1992).

[55] E. E. Flanagan, Phys. Rev. D 48, 2389 (1993).

[56] C. Cutler and M. Vallisneri, Phys. Rev. D 76, 104018 (2007).

[57] M. Vallisneri, Phys. Rev. D 77, 042001 (2008).

[58] E. Gustafson et al., LIGO Project Document Report No. T990080-00-D, 1999.

[59] In the actual calculation, we set $f_{g}=10^{-2} \mathrm{~Hz}$ just for convenience of numerical computation.

[60] The ET Science Team, Einstein Gravitational-Wave Telescope Conceptual Design Study, http://www.et-gw .eu/etdsdocument/, 2011.

[61] P. Amaro-Seoane, S. Aoudia, S. Babak, P. Binetruy, E. Berti et al., Classical Quantum Gravity 29, 124016 (2012).

[62] S. Kawamura, M. Ando, N. Seto, S. Sato, T. Nakamura et al., Classical Quantum Gravity 28, 094011 (2011).

[63] G. Harry, P. Fritschel, D. Shaddock, W. Folkner, and E. Phinney, Classical Quantum Gravity 23, 4887 (2006).

[64] B. C. Joshi, Int. J. Mod. Phys. D 22, 1341008 (2013). 